\title{
Ex-situ TEM: Insights into Metal Particle Growth During Fischer-Tropsch Synthesis
}

Chris Kliewer, Stu Soled and Gabor Kiss

ExxonMobil Research \& Engineering, Annandale, New Jersey, United States

Metal particle growth remains an important issue in catalysis. Reactor studies using Co-based FTS catalysts have revealed the existence of both rejuvenable ${ }^{1-5}$ and non-rejuvenable deactivation processes. 4,5 Given that Co has a melting point of $1768 \mathrm{~K}$ and a Tamman temperature of $\sim 0.55 \mathrm{Tm}$, the possibility of metal particle growth contributing to non-rejuvenable deactivation at FTS temperatures ( 473 - 493 K) became an important strategic question. Thus, detailed conventional and ex-situ TEM-based investigations were undertaken to understand the effect of FTS process conditions on small Co metal particles. 5

An experimental supported Co-based FTS catalyst was identified and prepared for conventional TEM studies by crushing it into fines using an agate mortar and pestle. The fines were dusted onto a holeycarbon coated TEM grid. In the case of the ex-situ study, fresh catalyst pellets were embedded in wax and ultramicrotomed in cross section. Electron transparent sections were collected onto a holey-carbon-coated TEM grid. The grid was transferred into a specially designed fixed bed reactor ${ }^{4-6}$ where it was treated and then transferred into the TEM via an inert protocol. ${ }^{4-6}$ Bright field (BF) TEM images were acquired from all materials, and in the case of the ex-situ study, the imaged areas were "mapped" so that the same metal particles could be re-examined subsequent to an additional ex-situ treatment.

Randomly-selected regions of spent catalyst from early (Figure 1a) and late (Figure 1b) in a pilot plant reactor run were examined first. Imaging clearly suggested that metal particle growth had occurred during FTS. However, because conventional TEM studies examine only small quantities of material and because it was impossible to rule out the existence of metal particle inhomogenieties within the fresh catalyst that might have led to the observed increase in particle size, an ex-situ TEM study was constructed to follow changes in the same catalyst particles with time on FTS and thus unequivocally determine whether or not cobalt metal particle growth was indeed occurring under actual process conditions.

Hence, the ex-situ reactor was packed with a fresh catalyst bed, and the TEM grid was placed near its outlet. The material was activated $\left(673 \mathrm{~K}, 1.2 \mathrm{MPa} \mathrm{H}_{2}, 8 \mathrm{~h}\right)$. Examination of the TEM grid immediately subsequent to the activation indicated the existence of small Co particles on the support (Figure 2a). The TEM grid was then returned to the reactor and run under FTS conditions (493 K, $2.0 \mathrm{MPa}$ syngas $\mathrm{H}_{2}$ : $\mathrm{CO}$ $\sim 2: 1$ ). The gas hourly space velocity (GHSV) was controlled to create $>90 \%$ CO conversion conditions. After 48 days on FTS, the TEM grid was discharged from the reactor. The same areas examined immediately subsequent to activation were re-examined. Metal particle growth was consistently observed throughout the catalyst (Figure 2b), confirming that metal particle agglomeration was indeed occurring at $493 \mathrm{~K}$ under FTS conditions. Further studies ultimately associated this low temperature agglomeration with a coalescence mechanism involving the hydrothermal oxidative environment created during FTS. ${ }^{4,5}$ 


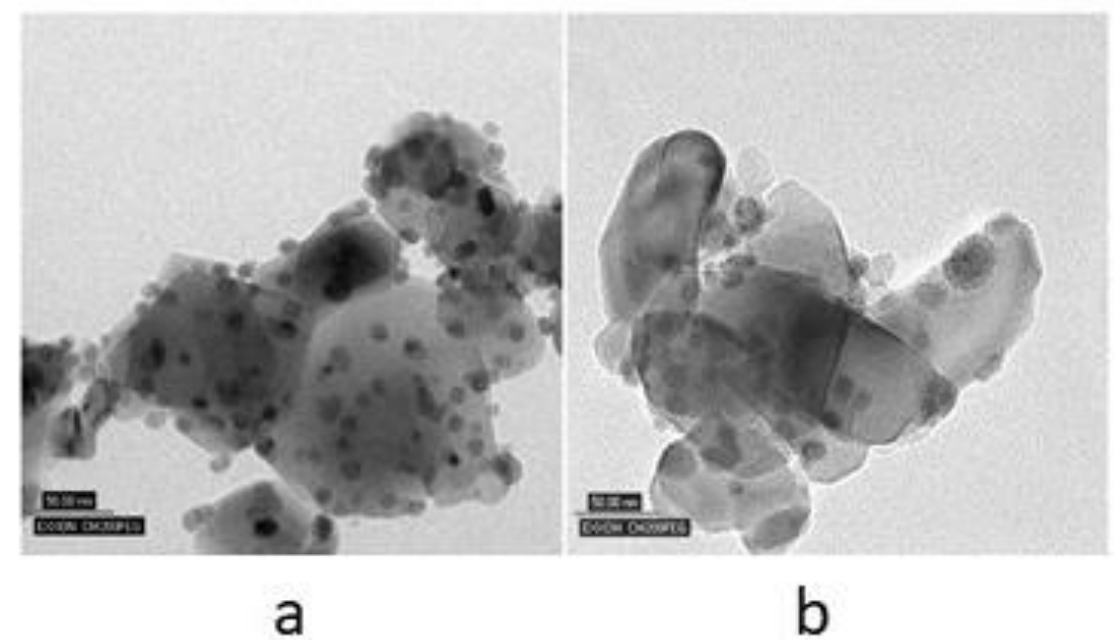

Figure 1. BF TEM image showing Co particles discharged: (a) early and (b) late in a pilot plant run.

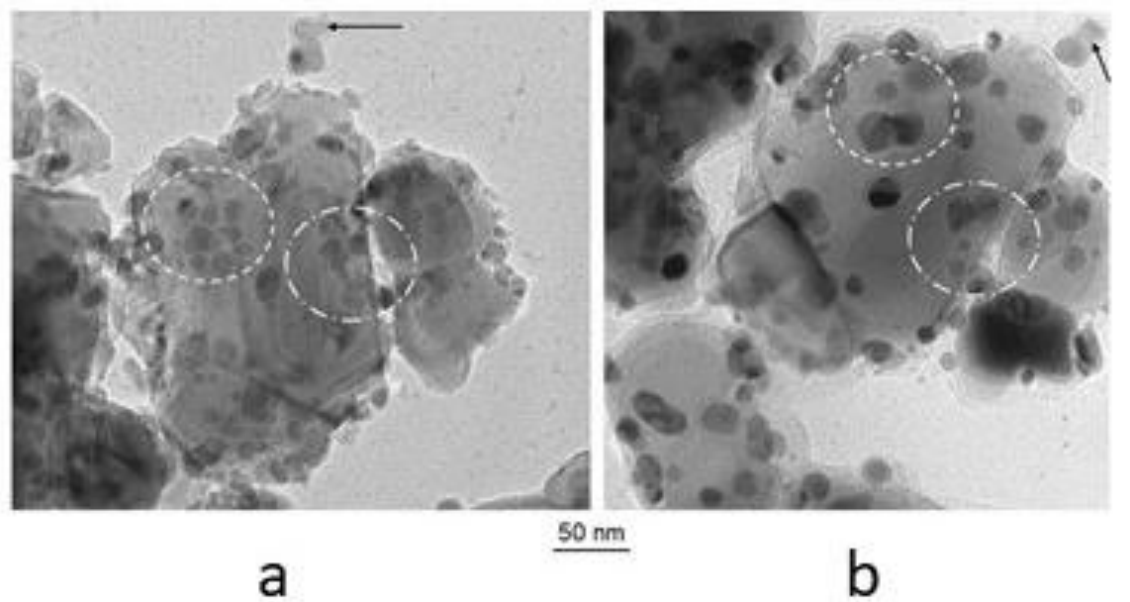

Figure 2. BF TEM image showing Co particles subsequent to: (a) activation and (b) 48 days on FTS in the ex-situ reactor. Circles and arrow indicate the same region in both images.

\section{References}

1. P.J. van Berge et. al. "Oxidation of Cobalt Based Fischer-Tropsch Catalysts as a Deactivation Mechanism" Catal. Today (2000) 58321.

2. J. Li et. al. "Fischer-Tropsch Synthesis: Effect of Water on the Deactivation of Pt Promoted $\mathrm{Co} / \mathrm{Al}_{2} \mathrm{O}_{3}$ Catalysts" Appl. Catal. A: General (2002) 228203.

3. G. Jacobs et. al. "Fischer-Tropsch Synthesis: Deactivation of Noble Metal-Promoted $\mathrm{Co} / \mathrm{Al}_{2} \mathrm{O}_{3}$ Catalysts" Appl. Catal. A: General (2002) 233215.

4. G. Kiss et. al. "Hydrothermal Deactivation of Silica-Supported Cobalt Catalysts in Fischer-Tropsch Synthesis" J. Catal. (2003) 217127.

5. C.E. Kliewer et. al. "Morphological Transformations During Fischer-Tropsch Synthesis on a TitaniaSupported Cobalt Catalyst” Catalysis Today (2019) 323233.

6. C.E. Kliewer et. al. "Ex-Situ Transmission Electron Microscopy: A Fixed Bed Reactor Approach" Microscopy and Microanalysis (2006) 12 (2) 135. 